UCB-PTH-04/34

LBNL-56671

NSF-KITP-04-129

SLAC-PUB-10879

\title{
String-Corrected Black Holes
}

\author{
Veronika E. Hubeny ${ }^{a}$, Alexander Maloney $^{b}$, And Mukund Rangamani ${ }^{a}$ \\ a Department of Physics, University of California, Berkeley, CA 94720, USA \\ Theoretical Physics Group, LBNL, Berkeley, CA 94720, USA \\ KITP, University of California, Santa Barbara, CA 93105, USA \\ ${ }^{b}$ SLAC and Department of Physics, Stanford University, Stanford, CA 94309
}

\begin{abstract}
We investigate the geometry of four dimensional black hole solutions in the presence of stringy higher curvature corrections to the low energy effective action. For certain supersymmetric two charge black holes these corrections drastically alter the causal structure of the solution, converting seemingly pathological null singularities into timelike singularities hidden behind a finite area horizon. We establish, analytically and numerically, that the string-corrected two-charge black hole metric has the same Penrose diagram as the extremal four-charge black hole. The higher derivative terms lead to another dramatic effect - the gravitational force exerted by a black hole on an inertial observer is no longer purely attractive! The magnitude of this effect is related to the size of the compactification manifold.
\end{abstract}

November 2004

veronika@berkeley.edu, maloney@slac.stanford.edu, mukund@socrates.berkeley.edu 


\section{Contents}

1. Introduction . . . . . . . . . . . . . . . . . . . . . . . . . . . . . . . . . 1

2. Review of Supersymmetric Attractor Mechanism . . . . . . . . . . . . . . . . 5

2.1. Basic Setup . . . . . . . . . . . . . . . . . . . . . . . . . 5

2.2. Black Hole Solutions . . . . . . . . . . . . . . . . . . . . . . . . . . . . 6

2.3. Calabi-Yau Black Holes . . . . . . . . . . . . . . . . . . . . . . . . . . 7

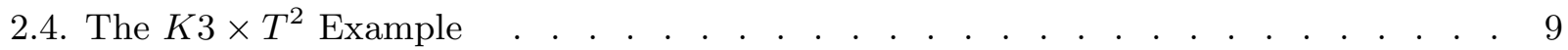

3. Black Hole Solutions: General Structure . . . . . . . . . . . . . . . . . . . . . 9

3.1. Leading Order Solutions . . . . . . . . . . . . . . . . . . . . . . . . . . 10

3.2. Asymptotic Behavior . . . . . . . . . . . . . . . . . . . . . . . . . . . . 11

3.3. Horizons and Singularities . . . . . . . . . . . . . . . . . . . . . . . . . . 13

4. Black Holes Solutions: Detailed Analysis . . . . . . . . . . . . . . . . . . . . . 13

4.1. Numerical Solutions . . . . . . . . . . . . . . . . . . . . . . . . . . . . 13

4.2. Non-Monotonic Metric Functions . . . . . . . . . . . . . . . . . . . . . . . 15

4.3. Causal Structure . . . . . . . . . . . . . . . . . . . . . . . . . . . . . . 17

5. Discussion . . . . . . . . . . . . . . . . . . . . . . . . . . . 18

\section{Introduction}

The physics of black holes has revealed many fascinating insights into the workings of quantum gravity. One of the most important observations, due to Bekenstein and Hawking, is that a black hole should be assigned an entropy equal to one quarter its horizon area,

$$
S=A / 4
$$

Strominger and Vafa showed that, for certain supersymmetric black holes, this entropy may derived microscopically in string theory [1]. They considered black holes with regular event horizons, whose areas are large in Planck units. This insight followed the remarkable paper of Sen [2], who studied four dimensional black hole solutions of heterotic string theory that classically have singular event horizons with zero area. The entropy of such black holes, which may be counted microscopically in string theory, is nevertheless non-zero. Sen conjectured that in this case string theoretic corrections lead to a finite area horizon, and used a brick wall model to reproduce the microscopic entropy up to a numerical coefficient. This idea, that quantum corrections should render sensible an apparently singular solution of general relativity has a rich history in string theory. In this paper we will consider a class of quantum corrections, which induce higher curvature terms quadratic in the Riemann tensor in the low energy effective action. For a wide class of black holes, these higher 
curvature corrections are sufficient to convert previously singular solutions into regular black holes with a finite area horizon.

Many authors have considered black hole solutions in the presence of higher curvature terms. In general one does not expect the relation between entropy and area to be of the Bekenstein-Hawking form (1.1), this being specific to the Einstein-Hilbert form of the Lagrangian. For generic higher derivative actions, Wald [3] has provided a formula for the macroscopic black hole entropy, generalizing the Bekenstein-Hawking relation (1.1). In a beautiful series of papers, the authors of [4,5] discussed supersymmetric black hole solutions in the presence of higher curvature terms. They showed that, for a class of black hole solutions including those of [1], this entropy formula correctly reproduces the subleading corrections to the microscopic entropy.1

Recently, Dabholkar [6] showed that these higher curvature corrections have a profound effect on the physics of the zero-horizon black holes considered by Sen. Remarkably, in this case one can reproduce an infinite series of subleading corrections to the entropy. Inspired by this, [7] showed that, for broad class of solutions that classically have zero horizon area, the area becomes non-zero once quantum corrections are taken into account. Thus quantum effects convert an apparently pathological solution of General Relativity into a regular black hole with an event horizon. For these black holes, higher curvature corrections to the Bekenstein-Hawking relation (1.1) are large - in this case, $S=A / 2$, where $A$ is the quantum corrected area.

All of the above derivations rely on the supersymmetric attractor mechanism [8], which states that the moduli of the compactification manifold approach fixed values at the horizon. This ensures that the entropy of a black hole depends only on the charges and not on the asymptotic values of the moduli. Moreover, it allows one to study near-horizon properties of a black hole without ever solving for an explicit metric.

In this note we will study the full geometry of these solutions, not just the near horizon behavior, and find the black hole metric including stringy higher-curvature corrections. We will consider the four dimensional effective theory found by compactifying type IIA on a

1 This result is all the more remarkable given that Wald's original derivation applies only to non-supersymmetric black holes. Wald's prescription assumes the existence of a bifurcate Killing horizon. Moreover, it is based on the first law of thermodynamics, which assumes a finite temperature. Neither of these conditions holds for supersymmetric black holes, so in this case Wald's formula must be regarded as a conjecture. However, given it's success in reproducing microscopic entropy formulae, it is an extremely well motivated one! 
Calabi-Yau three-fold. The resulting low energy action is $d=4, \mathcal{N}=2$ supergravity coupled to vector and hyper multiplets - the number of such multiplets and the form of the couplings depends on the choice of Calabi-Yau. The four dimensional supersymmetric black holes of this theory correspond to configurations of branes wrapping various cycles in the internal Calabi-Yau. The metric can then be found by solving a BPS equation, whose form in the presence of higher curvature corrections is quite complicated [5].

The prototypical example of this set-up is type IIA string theory compactified on $K 3 \times T^{2}$. In this case one can construct two-charge black holes by wrapping $Q_{4} \mathrm{D} 4$-branes on $K 3$, along with $Q_{0}$ D0-branes. This geometry is U-dual to the heterotic string black hole considered by [2], and also to the standard D1-D5 system. The microstates of this system may be counted exactly, leading to an entropy $S=4 \pi \sqrt{Q_{0} Q_{4}}$.

In the absence of higher curvature corrections, the four dimensional metric is

$$
d s^{2}=-e^{2 g} d t^{2}+e^{-2 g}\left(d r^{2}+r^{2} d \Omega_{2}^{2}\right),
$$

where

$$
e^{-2 g}=\sqrt{\left(1+\frac{Q_{0}}{r}\right)\left(1+\frac{Q_{4}}{r}\right)} .
$$

Near $r=0$, the metric becomes

$$
d s^{2}=-\rho^{2} d t^{2}+Q_{0} Q_{4}\left(4 d \rho^{2}+\rho^{2} d \Omega_{2}^{2}\right),
$$

where $r=\rho^{2} \sqrt{Q_{0} Q_{4}}$. This solution is singular at $r=\rho=0$, and has no event horizon. This is to be contrasted with the usual extremal Reissner-Nordstrom solution, which is of the form (1.2) with $e^{-2 g}=(1+Q / r)^{2}$. This solution has a finite horizon, with area $4 \pi Q^{2}$, and a near horizon $A d S_{2} \times S^{2}$ geometry. Typically, black holes with four or more charges are of the Reissner-Nordstrom type, and have a regular event horizon with finite area.

In string theory, both $\alpha^{\prime}$ and $g_{s}$ effects will lead to higher derivative terms in the low energy action. For type IIA strings on a Calabi-Yau, this includes terms of the form $F_{g} R^{2} W^{2 g-2}$, where $W$ is the graviphoton field strength and the $F_{g}$ depend on the Kahler moduli of the Calabi-Yau. In string perturbation theory, such couplings arise at the $g$ loop level. In the language of $\mathcal{N}=2$ supersymmetry, these couplings are F-terms, which can be efficiently computed in topological string theory - in fact, $F_{g}$ is simply the $g$-loop topological string partition function. This led the authors of [9] to conjecture a beautiful relation between topological string and black hole partition functions (see also [10]). 
We will consider cases where the internal Calabi-Yau is large in string units, so higher order $\alpha^{\prime}$ effects may be neglected. In this case the tree and loop level terms involving $F_{0}$ and $F_{1}$ are simple enough that the attractor equation can be solved explicitly. For $K 3 \times T^{2}$ the higher loop contributions $F_{g>1}$ vanish, although in general such terms will be present. In any case, once one has solved the attractor equations the metric is completely determined by a Killing spinor equation. As we will describe, this equation is a non-linear second order differential equation, which is generally difficult to solve. Nevertheless, we can learn from this equation many important features of the geometry. Moreover, it can be solved numerically. This allows us to incorporate the one-loop $g_{s}$ corrections to the black hole solutions in a controlled fashion.

We will show that once the higher curvature terms at one-loop are accounted for, the two-charge black hole is no longer described by the singular solution (1.2), (1.3). Instead, it is given by a regular black hole solution, with a timelike singularity hidden behind a finitearea event horizon. In many cases, the curvature of this horizon is large, so further $g_{s}$ and $\alpha^{\prime}$ corrections may be neglected. The causal structure of the solution is then identical to that of the four charge supersymmetric black hole spacetime. The near-horizon geometry thus becomes $A d S_{2} \times S^{2}$. Apart from these results, which might have been expected from the analysis of [7], we find some curious features. The metric component $g_{t t}$ is no longer a monotonic function of radius $r$. Instead, there are characteristic oscillations, whose period depends on the volume of some cycles in the internal Calabi-Yau. This implies that the gravitational potential is no longer purely attractive! An inertial observer could stay perched at a local minimum of the gravitational potential a finite, fixed distance from the black hole. This bizarre feature is due to the presence of higher derivative terms in the action which violate the energy conditions; in particular, we will see that the null energy condition is violated. It must be emphasized that there is a version of the positive energy theorem (PET) for these spacetimes by virtue of supersymmetry. Thus the total energy is positive definite, despite the presence of local pockets of negative energy density.

We begin in Section 2 with a brief summary of the generalized attractor mechanism in the presence of higher curvature interactions, as described by [4, 5]. In Section 3 we analyze the Killing spinor equations for a class of Calabi-Yau compactifications, and infer some general properties of the resulting black hole solutions. In Section 4 we study in detail the two charge supersymmetric black holes, before concluding in Section 5 . 2

2 While this paper was in preparation, reference [11] appeared, which discusses several similar 


\section{Review of Supersymmetric Attractor Mechanism}

In this section we review the construction of supersymmetric black hole solutions, including higher derivative $R^{2}$ corrections, given by [5]. This framework is appropriate for studying black holes geometries in $d=4, \mathcal{N}=2$ ungauged supergravity. As we describe in section 2.2, the Killing spinor equation for the metric in this case a second order differential equation. In section 2.3 we study this equation for Calabi-Yau compactifications of type IIA string theory, including tree level and one-loop contributions to the effective action. We discuss the special case of $K 3 \times T^{2}$ in 2.4 .

\subsection{Basic Setup}

Before delving into details, we will first review the construction of $\mathcal{N}=2$ supergravity actions with higher derivative terms. We will discuss here only a few salient details - a more complete review may be found in [12], and references therein.

We start with a gauged $\mathcal{N}=2$ superconformal theory in four dimensions. The field content of the theory, known as the Weyl multiplet, contains one gauge field for each generator of the $S O(4,2)$ superconformal group. This includes a veirbein and spin connection, which gauge translations and Lorentz transformations, as well as other fields that gauge dilatations, special conformal transformations and the various fermionic symmetries. This superconformal theory has the advantage that higher curvature terms are relatively easy to construct. To relate this to standard $\mathcal{N}=2$ supergravity, it is necessary to gauge fix the superconformal symmetry down to $\mathcal{N}=2$ Poincaré supersymmetry. This is achieved by the introduction of an extra multiplet, known as the conformal compensator, whose details will not be important for the current discussion. We will not review the details of the gauged superconformal theory, but instead simply quote the results as needed.

The low energy theory also includes $n_{v}+1$ vector multiplets, labeled by an index $I=0, \cdots, n_{v}$. One linear combination of these vector fields is the graviphoton, and the others are associated with deformations of the internal Calabi-Yau. The Weyl multiplet does not include the graviphoton, which gauges the central $U(1)$ of the theory, so we have included it explicitly as a vector multiplet. The lowest components of the vector multiplets are $n_{v}+1$ complex scalars $X^{I}$. In type IIA string theory, the $X^{I}$ are projective coordinates parameterizing the Kahler moduli space of an internal Calabi-Yau. The theory

issues for the $K 3 \times T^{2}$ black holes. 
also includes hypermultiplets, which are related to complex structure deformations of the Calabi-Yau, but these will not be important for our discussion.

The couplings between the vector and Weyl multiplets are summarized by a prepotential $F\left(X^{I}, W^{2}\right)$, where $W^{2}$ is the square of the graviphoton field strength. The function $F$ is holomorphic in the $X^{I}$ and obeys the homogeneity condition

$$
2 X^{I} F_{I}+W^{2} F_{W^{2}}=F
$$

Here $F_{I}$ and $F_{W^{2}}$ denote partial derivatives of $F$ with respect to $X^{I}$ and $W^{2}$, respectively.

When $F$ is independent of $W^{2}$, this is the usual holomorphic prepotential. When $F$ contains a term linear in $W^{2}$, the action includes curvature squared interactions. In general, we may expand the prepotential as

$$
F\left(X^{I}, W^{2}\right)=\sum_{g=0}^{\infty} F_{g}\left(X^{I}\right) W^{2 g} .
$$

In IIA string perturbation theory, the $g^{t h}$ term in this expansion appears as the $g$-loop level. To see this, recall that the dilaton, which determines the string coupling, lives in a hypermultiplet. The coupling between vector and hyper multiplets is highly constrained in $\mathcal{N}=2$ theories, completely fixing the dependence of the terms in (2.2) on $g_{s}$. The coefficients $F_{g}\left(X^{I}\right)$ are most readily computed in terms of a topological string partition function. We will write down a few explicit prepotentials in sections 2.3 and 2.4.

\subsection{Black Hole Solutions}

We will follow the notation used in [5], who discussed attractor and Killing spinor equations for stationary multi-center solutions. We will consider here only static, spherically symmetric black hole solutions of the form

$$
d s^{2}=-e^{2 g} d t^{2}+e^{-2 g}\left(d r^{2}+r^{2} d \Omega_{2}^{2}\right) .
$$

The electric and magnetic charges of the solution are denoted $p^{I}$ and $q_{I}$, where $I=0, \ldots, n_{v}$. The solution depends on these charges via the harmonic functions

$$
H^{I}(r)=h^{I}+\frac{p^{I}}{r}, \quad H_{I}(r)=h_{I}+\frac{q_{I}}{r}
$$

where $h^{I}$ and $h_{J}$ are constants related to the asymptotic values of the moduli $X^{I}$. 
The moduli $X^{I}$ will in general depend on $r$, and are determined in terms of the harmonic functions (2.4) by a set of stabilization equations [13, 14]. In terms of the rescaled variables

$$
\begin{aligned}
Y^{I}=e^{K / 2} \bar{Z} X^{I}, & \Upsilon=e^{K} \bar{Z}^{2} W^{2} \\
e^{-K}=i\left(\bar{X}^{I} F_{I}-\bar{F}_{I} X^{I}\right), & \left.Z=e^{K / 2}\left(p^{I} F_{I}-q_{I} X^{I}\right)\right)
\end{aligned}
$$

the stabilization equations are

$$
\begin{gathered}
Y^{I}-\bar{Y}^{I}=i H^{I} \\
F_{I}(Y, \Upsilon)-\bar{F}_{I}(\bar{Y}, \bar{\Upsilon})=i H_{I}
\end{gathered}
$$

In addition, the graviphoton field strength is fixed to be

$$
\Upsilon=-64\left(\nabla_{p} g\right)^{2}
$$

Having fixed the moduli, the metric is determined by a BPS equation, which takes the form [5]

$$
i\left[\bar{Y}^{I} F_{I}(Y, \Upsilon)-\bar{F}_{I}(\bar{Y}, \bar{\Upsilon}) Y^{I}\right]-e^{-2 g}=128 i e^{g} \nabla^{p}\left[\left(\nabla_{p} e^{-g}\right)\left(F_{\Upsilon}-\bar{F}_{\Upsilon}\right)\right]
$$

The indices $p, q$ label the three flat spatial directions.

\subsection{Calabi-Yau Black Holes}

For type IIA string theory on a generic Calabi-Yau, the number of vector multiplets $n_{v}$ is fixed by the topology of the Calabi-Yau - roughly, it counts the number of fourcycles in the Calabi-Yau. The moduli $Y^{I}$ then measure the sizes of these cycles. When the Calabi-Yau is large, so that $\alpha^{\prime}$ corrections may be neglected, the first two terms in the pre-potential are

$$
F\left(Y^{I}, \Upsilon\right)=D_{A B C} \frac{Y^{A} Y^{B} Y^{C}}{Y^{0}}+\frac{d_{A} Y^{A}}{Y^{0}} \Upsilon
$$

Here $D_{A B C}$ is related to the intersection numbers and $d_{A}$ is given by the second Chern class of the Calabi-Yau dotted into the various four-cycles. The constraint that $\alpha^{\prime}$ corrections are small requires that certain ratios of the charges be taken large.

3 The authors of [5] included a constant $\chi$ in their discussion of the BPS equations, which sets the normalization of the Einstein-Hilbert term in the Lagrangian. We have set here $\chi=-2$. 
For this prepotential the attractor equations (2.6) can be solved exactly if we take $H^{0}=0$. To this end, we define $D_{A B}=D_{A B C} H^{C}$ and its inverse $D_{A B} D^{B C}=\delta_{A}^{C}$. Then $\mathrm{\theta}$

$$
\begin{aligned}
Y^{A} & =\frac{i}{2} H^{A}+\frac{1}{6} Y^{0} D^{A B} H_{B}, \\
Y^{0} & =\sqrt{\frac{\frac{1}{4} D-d_{A} H^{A} \Upsilon}{H_{0}+\frac{1}{12} D^{A B} H_{A} H_{B}}}
\end{aligned}
$$

where

$$
D=D_{A B C} H^{A} H^{B} H^{C} .
$$

When $D_{A B}$ is not invertible, the matrix $D^{A B}$ in these expressions is replaced by the pseudo-inverse of $D_{A B}$.

From (2.10), it follows that

$$
\begin{aligned}
i\left[\bar{Y}^{I} F_{I}-Y^{I} \bar{F}_{I}\right] & =-\frac{D}{Y^{0}}+2 \Upsilon \frac{d_{A} H^{A}}{Y^{0}} \\
F_{\Upsilon}-\bar{F}_{\Upsilon} & =i \frac{d_{A} H^{A}}{Y^{0}} .
\end{aligned}
$$

If we define

$$
\begin{aligned}
& a(r)=H_{0}+\frac{1}{12} H_{A} D^{A B} H_{B}, \\
& b(r)=64 d_{A} H^{A}, \\
& d(r)=D=D_{A B C} H^{A} H^{B} H^{C},
\end{aligned}
$$

then, using (2.7), and (2.10), we find

$$
Y^{0}=\sqrt{\frac{d(r)+4 g^{\prime}(r)^{2} b(r)}{4 a(r)}} .
$$

In addition, introducing

$$
\xi(r)=\frac{b(r)}{Y^{0}}
$$

we can write (2.8) as

$$
e^{-2 g(r)}=\frac{d(r)}{Y^{0}}+2\left[\xi(r)\left(g^{\prime \prime}(r)+\frac{2}{r} g^{\prime}(r)\right)+\xi^{\prime}(r) g^{\prime}(r)\right] .
$$

While we have reduced the problem of finding the metric to solving a differential equation, this is still a fairly complicated non-linear problem.

4 We are using the fact that 2.7) implies that $\Upsilon$ is real. For stationary solutions this is no longer guaranteed. 


\subsection{The $K 3 \times T^{2}$ Example}

Let us now consider a special example, where the Calabi-Yau is $K 3 \times T^{2}$. In this case the resulting low energy theory actually has $\mathcal{N}=4$ supersymmetry, but we will continue to use the use the $\mathcal{N}=2$ language. There are now 24 vector multiplets, corresponding to the two cycle of $T^{2}$, the 22 two-cycles of K3, and the graviphoton. The prepotential takes the form

$$
F\left(Y^{I}, \Upsilon\right)=-\left(\frac{Y^{1}}{Y^{0}}\right)\left(\frac{1}{2} C_{\alpha \beta} Y^{\alpha} Y^{\beta}+\frac{1}{64} \Upsilon\right)
$$

where $\alpha, \beta=2, \cdots 23$ label the two-cycles of $\mathrm{K} 3$ and $C_{\alpha \beta}$ is the intersection matrix of K3. For a general Calabi-Yau there will be loop corrections of order $\Upsilon^{2}$ and higher to the prepotential. However, for $K 3 \times T^{2}$ these additional contributions vanish and the prepotential has only tree-level and one loop contributions. This full, quantum corrected prepotential can be found exactly (see e.g. Eq. (19) of [6]), although we will focus only on terms that are tree level in $\alpha^{\prime}$, which are given by (2.17).

We are interested in black hole solutions corresponding to the D0-D4 systems discussed in the introduction. For these black holes, it is sufficient to take

$$
H^{1}=1+\frac{Q_{4}}{r}, \quad H_{0}=1+\frac{Q_{0}}{r}, \quad H^{a}=h^{a}, \quad H_{a}=H^{0}=0 .
$$

If we define

$$
\begin{aligned}
Y^{0} & =\sqrt{\frac{\beta}{4}+g^{\prime 2}} \sqrt{\frac{H^{1}}{H_{0}}}, \\
\beta & =\frac{1}{2} C_{a b} h^{a} h^{b}, \\
\zeta(r) & =\frac{H^{1}}{Y^{0}}=\sqrt{H^{1} H_{0}} \frac{1}{\sqrt{\frac{\beta}{4}+g^{\prime 2}}},
\end{aligned}
$$

then the equation of motion for the metric is

$$
e^{-2 g}=\beta \zeta+2\left[\zeta\left(g^{\prime \prime}+2 \frac{g^{\prime}}{r}\right)+\zeta^{\prime} g^{\prime}\right] .
$$

Solutions to (2.20) then describe $g_{s}$-corrected black hole geometries.

\section{Black Hole Solutions: General Structure}

In this section we study the solutions to the Killing spinor equation (2.16). The solutions are difficult to find analytically, but we will see that nevertheless one can extract 
the causal structure. We will consider a broad class of Calabi-Yau black holes, which classically (i.e., without $R^{2}$ corrections) have null singularities and zero horizon area. We show that once higher curvature corrections are added, the horizon becomes non-singular and the causal structure is identical to that of the familiar extremal Reissner-Nordstrom solution.

\subsection{Leading Order Solutions}

First, we review the solutions in the absence of $R^{2}$ corrections. The $g^{\prime \prime}(r)$ and $g^{\prime}(r)$ terms drop out of the Killing spinor equation (2.16), and the warp factor $e^{-2 g}$ in the metric is determined algebraically:

$$
e^{-2 g}=2 \sqrt{d(r) a(r)}
$$

At infinity the solution is asymptotically flat, with

$$
e^{2 g} \rightarrow 1, \quad r \rightarrow \infty
$$

provided we adjust the asymptotic values of the moduli $\left(h^{A}, h_{A}\right)$ appropriately. When a sufficient number of magnetic charges $p^{A}$ are non zero, so that $D_{A B C} p^{A} p^{B} p^{C} \neq 0$, the solution has the form

$$
e^{2 g} \rightarrow\left(\frac{r}{R_{0}}\right)^{2}, \quad r \rightarrow 0
$$

near the origin. Here

$$
R_{0}=\sqrt{D_{A B C} p^{A} p^{B} p^{C} \hat{q}_{0}}
$$

where $\hat{q}_{0}=q_{0}+\frac{1}{12} \tilde{D}^{A B} q_{A} q_{B}$, with $\tilde{D}^{A B}$ the (pseudo) inverse of $D_{A B C} p^{C}$. When $R_{0} \neq$ 0 the geometry has a non-singular event horizon of area $4 \pi R_{0}^{2}$, and the near horizon geometry is $A d S_{2} \times S^{2}$.

For the rest of this paper, we will consider solutions with $D_{A B C} p^{A} p^{B} p^{C}=0$. In this case the radius (3.4) vanishes and the function $e^{2 g}$ vanishes more slowly as $r \rightarrow 0$. In particular,

$$
e^{2 g} \sim r^{\alpha}, \quad r \rightarrow 0
$$

where $\alpha$ is $\frac{1}{2}$, 1 , or $\frac{3}{2}$ depending on the number of non-vanishing magnetic charges. For these solutions the curvature diverges as $r \rightarrow 0$. For the D0-D4 system on $K 3 \times T^{2}, \alpha=1$, as can be seen from (1.2). In any case, the curvature singularity is null, and coincides with the horizon at $r=0$. The Penrose diagram for these black holes is shown in Fig.1. We will now move on to the $R^{2}$ corrected metric. 


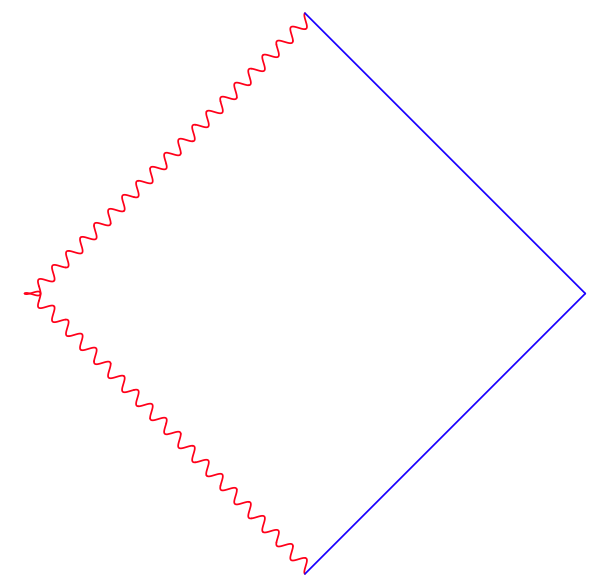

Fig. 1: Penrose diagram for the classical black hole with vanishing horizon area.

\subsection{Asymptotic Behavior}

Before delving into details, we will first consider the asymptotic behavior of the solutions to the Killing spinor equation (2.16).

At $r \rightarrow \infty$, the differential equation (2.16) simplifies considerably. We have

$$
e^{-2 g} \rightarrow 2 \sqrt{a(r) d(r)}
$$

plus corrections at $\mathcal{O}\left(r^{-3}\right)$. This goes to a constant out at infinity, indicating that the space is asymptotically flat. Moreover, by an appropriate choice of the asymptotic values of the moduli $\left(h_{A}, h^{A}\right)$ we can ensure that $e^{2 g} \rightarrow 1$. Of course, this is provided that $D_{A B C} h^{A} h^{B} h^{C}$ and $h_{0}+\frac{1}{12} D^{A B} h_{A} h_{B}$ are non-zero. As is clear from (3.6), we will find a non-asymptotically flat geometry when either of these quantities vanish. For instance, consider the simple case

$$
H^{A} \equiv 0, \forall a \neq 1, \quad \text { and } \quad H^{1} \neq 0
$$

Then from (2.13) and (2.14) we find that

$$
d(r)=0, \quad \text { and } \quad Y^{0}=g^{\prime}(r) \sqrt{\frac{b(r)}{a(r)}} .
$$

It is easy to show that (2.16) reduces for this special case to

$$
e^{-2 g}=2\left(\frac{d}{d r} \sqrt{a(r) b(r)}+\frac{2}{r} \sqrt{a(r) b(r)}\right) .
$$


This implies that $e^{-2 g} \rightarrow c / r$, with $c=4 \sqrt{a_{\infty} b_{\infty}}$, for large $r$. The metric is not asymptotically flat. 5 To understand this, note that (3.7) implies that at large $r$ we are driven towards a singular point in the Calabi-Yau moduli space. In particular, as $r \rightarrow \infty$ some of the ratios $Y^{A} / Y^{0}$, which measure sizes of cycles in the internal Calabi-Yau, become small. Thus $\alpha^{\prime}$ effects can no longer be neglected. This effect is seen from the four dimensional perspective as a non-asymptotically flat geometry. For the rest of the paper we will ensure that this does not happen by choosing constants $\left(h^{A}, h_{A}\right)$ such that $a(r)$ and $d(r)$ are non-zero at large $r$.

Let us now consider the metric at $r \rightarrow 0$. Once higher curvature corrections are taken into account,

$$
e^{2 g} \rightarrow\left(\frac{r}{R_{0}}\right)^{2}, \quad r \rightarrow 0,
$$

so the near horizon geometry is $A d S_{2} \times S^{2}$, with finite radius $R_{0}$. To see this, note that with the ansatz (3.10) the Killing spinor equation (2.16) becomes

$$
e^{-2 g}=\frac{2}{r^{2}} \sqrt{d_{A} p^{A} \hat{q}_{0}}
$$

plus sub-leading corrections of order $\mathcal{O}(r)$. 6 This implies that the solution takes the form (3.10), provided

$$
R_{0}=\sqrt{2}\left(d_{A} p^{A} \hat{q}_{0}\right)^{1 / 4}
$$

is non-zero. This corresponds to a horizon area

$$
A=\left.4 \pi e^{-2 g(r)} r^{2}\right|_{r=0}=8 \pi \sqrt{d_{A} p^{A} \hat{q}_{0}},
$$

which agrees with the result of [7]. Note that we could have concluded this earlier, from (3.9). Even though the choice of moduli (3.7) leads to problems in the asymptotic form of the metric, the supersymmetric attractor mechanism guarantees that the near horizon physics is independent of this asymptotic behavior. Typically, by taking $q_{0}, p^{A} \gg 1$ this area can be made macroscopic, i.e.much larger than the string or Planck scale.

5 Strangely enough, the asymptotic metric in this case looks like the singular near-horizon metric (1.4). We should remark here that there is no singularity in this case. The geometry (1.4) is singular only as $r \rightarrow 0$; for large $r$ it is a geodesically complete spacetime.

${ }^{6}$ We have used the fact that, since we are considering solutions with $D_{A B C} p^{A} p^{B} p^{C}=0$, the function $D / Y^{0}$ may be neglected as $r \rightarrow 0$.

7 We should emphasize that this holds only for the generic case - for $K 3 \times T^{2}$, the string coupling is determined by the charges (due to the enhanced $\mathcal{N}=4$ supersymmetry). In this case, the resulting horizon is string scale. 


\subsection{Horizons and Singularities}

Having understood the asymptotic behavior of the solution, we can now ask what happens in the intermediate region where $r$ is finite and non-zero. The Killing spinor equation is quite difficult to solve analytically, but we may nevertheless establish several results concerning the causal structure of the solution.

First, we would like to ask whether the $R^{2}$ corrections lead to additional singularities or horizons at finite $r$. In the parameterization (2.3), these correspond to points where $e^{2 g}$ diverges or vanishes at finite values of $r \neq 0$. We will now demonstrate that this is not the case, and that the metric is smooth and non-degenerate for all positive values of $r$.

Let us assume was have such a point, where $|g| \rightarrow \infty$ at a finite, positive value of $r=r_{o}$. At this point, both $\left|g^{\prime}(r)\right|$ and $Y_{0} \sim\left|g^{\prime}(r)\right|$ diverge. The Killing equation (2.16) then implies that

$$
e^{-2 g}=\frac{1}{8}\left(\frac{2}{r}+\partial_{r}\right) \sqrt{b(r) a(r)} .
$$

It is straightforward to see that the right hand side is finite and non-zero for any finite $r_{o}$. This contradicts our assumption that $|g| \rightarrow \infty$, so we conclude that the solution is non-singular and non-degenerate.

\section{Black Holes Solutions: Detailed Analysis}

We now turn to a detailed analysis of (2.16), which as we have seen is difficult to treat

analytically. 8 We will see that the numerical solutions provide a good deal of information about the spacetime and are quite robust.

\subsection{Numerical Solutions}

In this section we describe numerical solutions to the Killing spinor equation (2.16), which verify the analytic arguments of the previous section. For simplicity, we will consider the case where all but one of the magnetic charges vanishes. This includes the two-charge black hole configurations on $K 3 \times T^{2}$, mentioned earlier and the subject of [6].

8 One could consider series solutions to (2.16); however, expanding $e^{-2 g}$ in a power series around the origin or around $r=\infty$, we find that successive series terms tend to lower the radius of convergence. One possibility is to resum the resulting series and analytically continue beyond the domain of convergence, obtaining a closed form solution. Unfortunately, this method proves too complicated, so we turn to numerical solutions. 

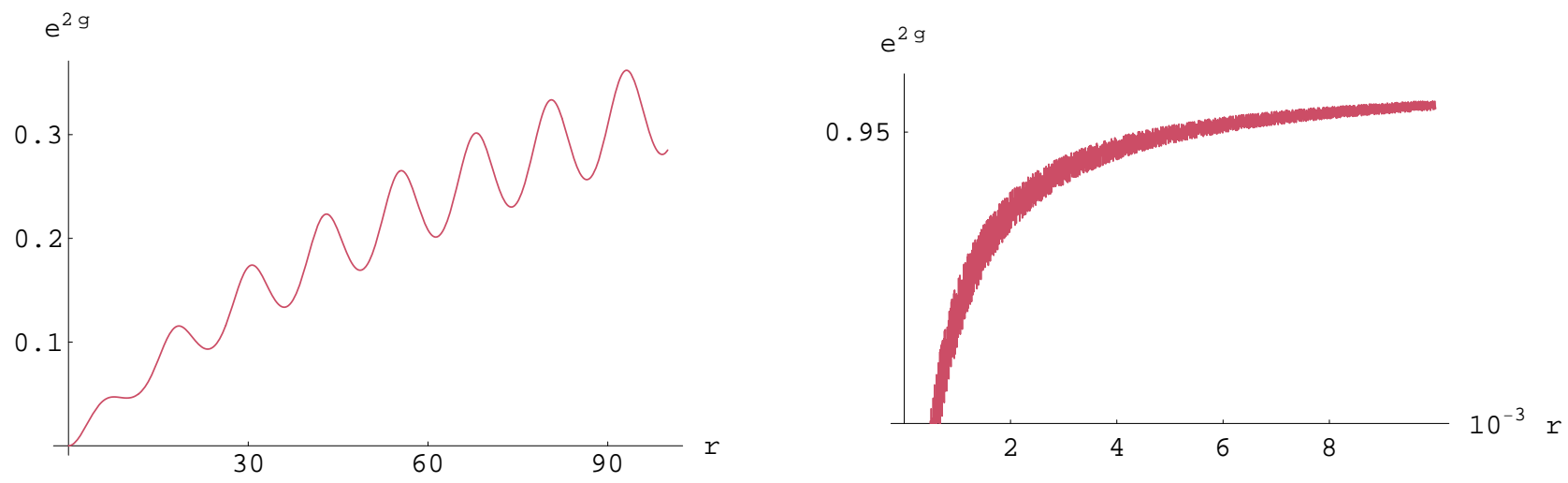

Fig. 2: Plot of the function $e^{2 g}$ for $Q_{0}=500$ and $Q_{4}=60$. The first plot shows the behavior of the function for small $r$, and the second plot the behavior at large $r$.

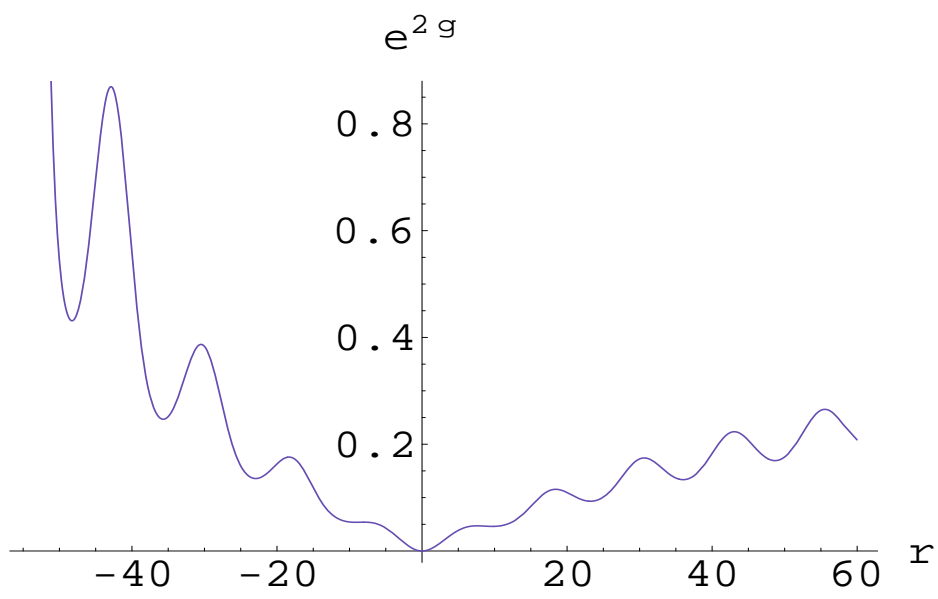

Fig. 3: Plot of the function $e^{2 g}$ for $Q_{0}=500$ and $Q_{4}=60$. The divergence of the function as $r \rightarrow-Q_{4}$ is indicative of a singularity.

In this case, one can find a constant $\gamma$ such that

$$
d(r)=\gamma^{2} d_{A} H^{A} \Rightarrow Y^{0}=\sqrt{\frac{\gamma^{2}}{256}+g^{\prime 2}} \sqrt{\frac{b(r)}{a(r)}},
$$

and the equations of motion simplify considerably. In fact, it is easy to see that (2.16) can be written in the form $(2.20)$, with $\gamma^{2}=64 \beta$. We will henceforth concentrate on equation (2.20), with the various parameters as defined in (2.18), (2.19). This allows us to make contact with (1.2) at various stages of the analysis.

First, note that for an asymptotically flat solution, we need to choose $\beta=1 / 4$. This ensures that the metric is correctly normalized at infinity, i.e., $e^{2 g} \rightarrow 1$. In Fig.2, we show 
the numerical solution for the case $Q_{0}=500$ and $Q_{4}=60$. In Fig.3, we extend the same solution to negative values of $r$ - this is the region behind the horizon. One can find such solutions for any choice of $\left(Q_{0}, Q_{4}, \beta\right)$. In determining the boundary conditions for the numerical evolution we make use of the series solution around $r=0$ to set the value of the $g(r)$ and $g^{\prime}(r)$. As can be seen from the plots Fig.2, this solution satisfies the required near-horizon and asymptotic behavior, viz., $\mathrm{AdS}_{2} \times S^{2}$ and asymptotically flat (since the oscillations die out at infinity).

\subsection{Non-Monotonic Metric Functions}

The most striking feature of the solutions Fig.2 and Fig.3 is that the metric function $e^{2 g}$ is not monotonic. 9 This is in marked contrast with the leading order solution (1.2). One can check numerically that the period of the oscillations is independent of the charges. In fact, the oscillation frequency $\omega$ is given by 10

$$
\omega=\sqrt{\beta},
$$

so that the separation between local maxima is $2 \pi / \sqrt{\beta}$. Note that the characteristic oscillation period is set by the volume of some cycle of the Calabi-Yau at large $r$. For example, it is easy to check that $Y^{1} / Y^{0} \rightarrow 2 / \sqrt{\beta}$ as $r \rightarrow \infty$, for this class of examples.

The main effect of the non-monotone metric function is that there are inertial observers, who can sit at a fixed constant distance away from the black hole; the gravitational force felt by an inertial observer will not be universally attractive towards the origin. Instead, there will be small regions where the force is repulsive. To see this, note that the function $e^{2 g}$ is the gravitational potential felt by a timelike geodesic. In particular, timelike radial geodesics, parameterized by an affine parameter $s$, are given by

$$
\dot{r}^{2}=E^{2}-e^{2 g}, \quad t(s)=E \int^{s} e^{-2 g\left(r\left(s^{\prime}\right)\right)} d s^{\prime}
$$

9 Curiously, integrating in from $r=\infty$ with appropriate boundary conditions, $e^{2 g}$ is monotonic but encounters a singularity at some $r>0$. Since we are interested in solutions with a regular horizon we discard this solution as unphysical.

10 To understand this feature analytically, note that when $g^{\prime}$ is small, the equation of motion (2.20) becomes

$$
\left(\partial_{r}^{2}+\beta\right) e^{2 g}=\zeta^{-1}
$$

Thus in this regime the $e^{2 g}$ takes the form of a homogeneous solution, which is periodic with frequency $\sqrt{\beta}$, plus a non-periodic particular solution. 

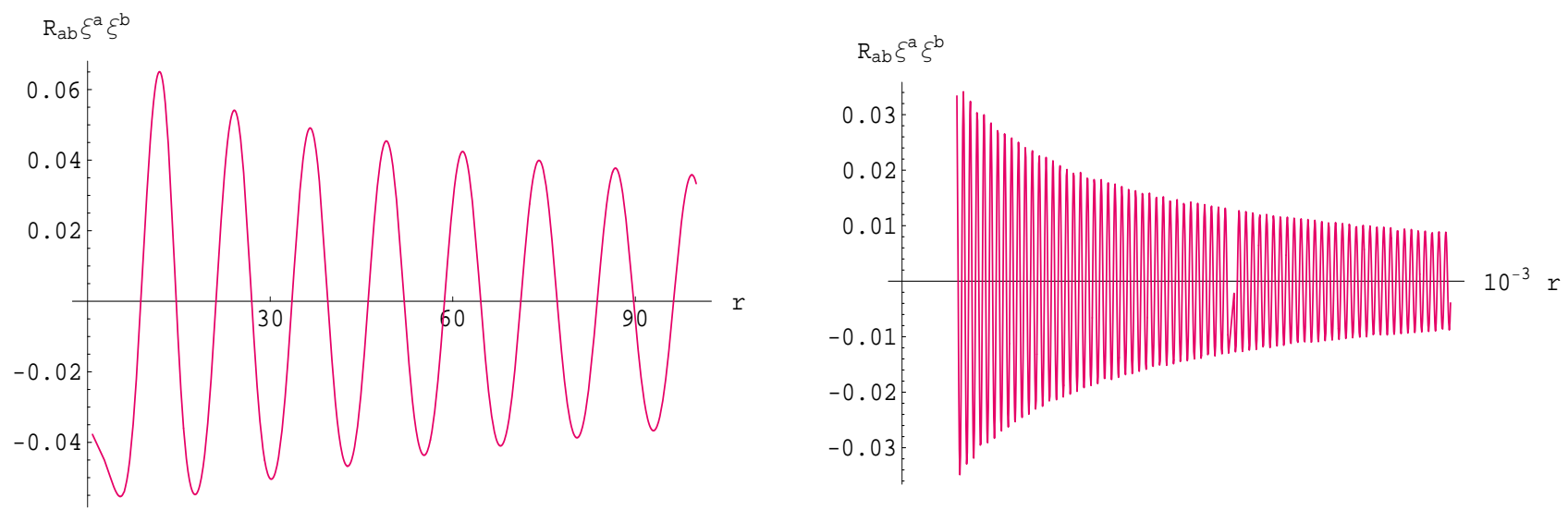

Fig. 4: Plot of $R_{\mu \nu} \xi^{\mu} \xi^{\nu}$ to demonstrate failure of the null energy condition. One again we choose $Q_{0}=500$ and $Q_{4}=60 . \xi^{\mu}$ is chosen to be the null vector tangent to radial null geodesics.

where $E$ is a constant of motion. The dynamics are simply that of a particle of energy $E^{2}$ moving in a potential $e^{2 g}$. An inertial observer may then sit at a finite value of $r$ where $e^{2 g}$ is at a local minimum, where the attractive and repulsive gravitational forces are balanced.

The reason for the bizarre behavior can be traced to the fact that the $R^{2}$ terms violate energy conditions. In this event, it is possible to have local pockets of negative energy density, which will manifest themselves in the form of repulsive gravitational force. Given the numerical solution for the metric, it is easy to check this violation of energy conditions. Of course, since we have a higher derivative action, it is not clear how we should define the stress tensor. One option is to incorporate the higher derivative terms as explicit contributions to the stress tensor, writing the equation of motion schematically as $G_{\mu \nu}=8 \pi T_{\mu \nu}^{c}$, where $T_{\mu \nu}^{c}$ includes both the matter and the higher derivative gravitational contributions. With this definition it is easy to define the energy conditions in the conventional fashion. For example, the null energy condition will require that $R_{\mu \nu} \xi^{\mu} \xi^{\nu} \geq 0$ for any null vector $\xi^{\mu}$. We show that this is not satisfied in the present example, by exhibiting a null vector for which $R_{\mu \nu} \xi^{\mu} \xi^{\nu}$ is not positive definite in Fig.4. It suffices to take $\xi^{\mu}$ to be the null vector tangent to radial null geodesics in the $(r, t)$ plane, i.e., $\xi=\partial_{t}+f(r) \partial_{r}$. At the same time the supersymmetric nature of the solution guarantees us that these backgrounds respect the positive energy theorem (PET). This however only implies that the total energy of any excitation in the system is bounded from below by zero, with the supersymmetric black hole being the ground state. Since this is only a statement about the total energy as 
measured by an asymptotic observer, there is no contradiction with the presence of local pockets of negative energy.

We note in passing that, due to the violation of the energy conditions, the null convergence condition does not obviously follow from the Raychaudhuri equation. However, our spacetime satisfies the Averaged Null Energy Condition (ANEC), which requires that along every affinely parameterized inextendible null geodesic in the spacetime with tangent $\xi^{a}$ and affine parameter $\lambda$,

$$
\int d \lambda R_{\mu \nu} \xi^{\mu} \xi^{\nu} \geq 0
$$

It can be shown that the focusing theorems are satisfied with the weakened ANEC [15]. This, in particular, should ensure that the area theorem holds for our spacetime. In fact, since the relation between entropy and area is $S=A / 2$ [7], in order for the second law of thermodynamics to be satisfied it is clear that the area theorem must be true.

\subsection{Causal Structure}

We will now investigate the causal structure of the higher-curvature corrected solution. The causal structure is fixed completely by the radial null geodesics, which follow trajectories $t(r)$ given by

$$
t^{ \pm}(r)= \pm \int^{r} e^{-2 g\left(r^{\prime}\right)} d r^{\prime}
$$

Here $t^{+}$and $t^{-}$denote ingoing and outgoing geodesics. Since the integrand is strictly positive and finite, $t^{ \pm}(r)$ are strictly monotone and continuous functions of $r$. Moreover, we established that near $r=0$ and $r=\infty$ the form of $e^{-2 g}$ is, up to overall constants, precisely that of the four charge black hole. These two results are sufficient to establish that these two geometries have precisely the same causal structure outside the horizon.

From the full numerical solution we can verify concretely that the causal structure of the string-corrected two charge black holes is identical to that of the four charge supersymmetric black hole. First of all, note that the metric function $e^{2 g}$ is positive definite and finite for $r>0$. So the $(r, t)$ coordinates in (2.3) are a good coordinate chart for $r>0$, with $\frac{\partial}{\partial t}$ being a timelike Killing vector.

The coordinate chart breaks down at $r=0$, where the Killing vector $\frac{\partial}{\partial t}$ becomes null. A non-degenerate coordinate chart can be introduced following the standard route for constructing ingoing/outgoing Eddington coordinates: $u, v=t \mp \int^{r} e^{-2 g\left(r^{\prime}\right)} d r^{\prime}$. All we learn from the exercise is that the surface $r=0$ is a null surface, which can be thought of 


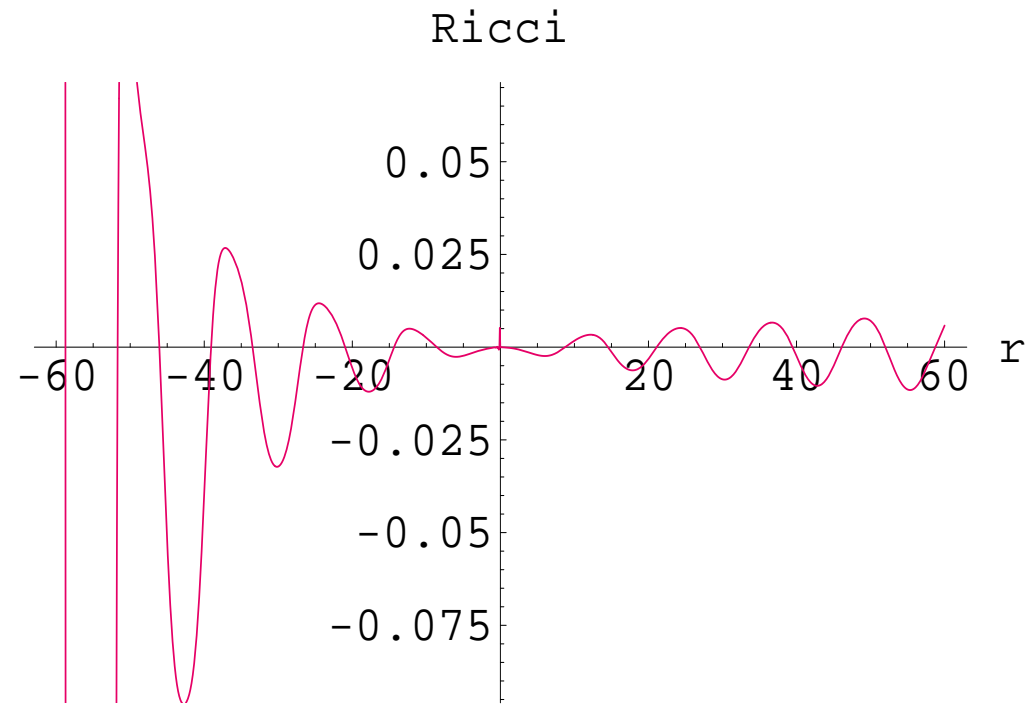

Fig. 5: Plot of the Ricci scalar for $Q_{0}=500$ and $Q_{4}=60$. The divergence as $r \rightarrow-Q_{4}$ indicates the location of the timelike singularity.

as the Poincare horizon of the Bertotti-Robinson spacetime. Since this is just a coordinate singularity, we can continue to the region $r<0$. Once again we find that the function $e^{2 g}$ is positive definite, and hence $\frac{\partial}{\partial t}$ is a timelike Killing vector. However, we find that (we are assuming that $Q_{0}>Q_{4}$, as is required to suppress $\alpha^{\prime}$ corrections to the pre-potential)

$$
e^{2 g} \rightarrow \infty, \quad r \rightarrow-Q_{4},
$$

implying that there is a singularity. One can indeed check this explicitly by considering the curvature invariants; for example, the Ricci scalar blows up as we approach $r=-Q_{4}$ as can be seen in Fig.5.

We conclude that the class of quantum corrections considered in this paper convert null singular spacetimes to regular black hole geometries. The Penrose diagram of this spacetime is therefore as shown in Fig.6.

\section{Discussion}

We have considered supersymmetric black holes in four dimensions that arise in string theory compactified on a Calabi-Yau. Our focus has been on solutions which seem to have singular event horizons, when viewed as solutions to the low energy effective $\mathcal{N}=2$ supergravity theory. Quantum effects, in the form of higher derivative corrections to the Einstein-Hilbert action, serve to smooth out the horizon to a regular null hypersurface. 


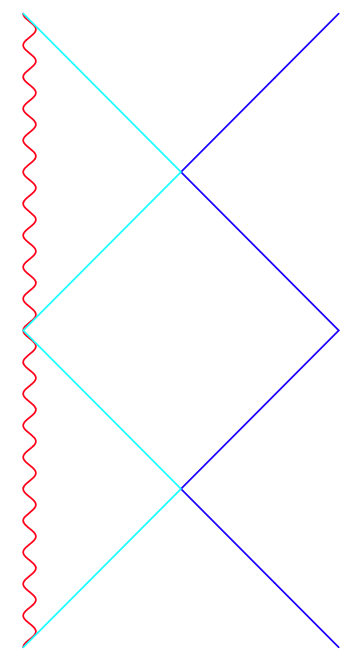

Fig. 6: Penrose diagram for the quantum corrected black hole.

This result is valid for a large class of black holes which arise in Calabi-Yau compactifications. Our main result is that the causal structure of the quantum corrected solution is identical to that of the four charge supersymmetric black hole in four dimensions.

The quantum corrected metric exhibits a very unusual feature: the metric function is non-monotonic outside the horizon. To the best of our knowledge, this is the first example of a black hole with this feature. As a result, these black hole solutions admit inertial observers who can passively view the black hole from a fixed finite distance. This is possible because local pockets of negative energy are allowed by virtue of higher derivative terms which violate an energy condition. Furthermore, we have demonstrated that the spacetime satisfies the Averaged Null Energy Condition, which is sufficient to prove the area theorem in the present context.

At this point we should ask to what extent our approximations are justified. We have incorporated the one-loop correction to the pre-potential, but have otherwise been working at tree level in $\alpha^{\prime}$. This is justified when we can take the Calabi-Yau to be large. However, the attractor mechanism does not always accord us this luxury, since it fixes the near-horizon behavior of the moduli. In the simplest case of $K 3 \times T^{2}$, the size of the horizon is large in four dimensional Planck units, provided we choose the charges to be large. However, translating to string units, we find that the size is string scale, because the dilaton is fixed in terms of the charges by virtue of the $\mathcal{N}=4$ supersymmetry. To see this, note that the D0-D4 system is U-dual to a fundamental heterotic string with momentum 
and winding proportional to the charges. Since we require that the supergravity solution reproduce the entropy of the fundamental string configuration, we are forced to be at the correspondence point [16], where the horizon size is string scale.

For generic Calabi-Yau black holes, $g_{s}$ is not constrained by the charges, so by taking the charges to be large the horizon area can be made large in both string and Planck units. However, in this case there is another effect which may be relevant - as one increases the charges, the size of some cycles in the Calabi-Yau will grow. In general, one might therefore worry that there will be corrections due to Kaluza-Klein modes. Moreover, we would like to have a quantitative understanding of higher derivative terms which are not of the specific type considered here. Although in these cases the resulting equations are much more complicated, we hope to report on this in the near future.

Note added: In [11], it was pointed out that the oscillations in the numerical solution to (2.16), do not die out rapidly enough as $r \rightarrow \infty$. One can check that the asymptotic behaviour of the numerical solution is $e^{2 g} \sim \cos (\beta r) / r$, which resembles a propagating mode. It was suggested in [11] that an appropriate field redefinition be done to remove this effect as the propagating mode is a ghost mode, arising as a result of the field equations being higher order in derivatives. A field redefinition was suggested based on linearised analysis about flat space. We note that such a field redefinition will indeed change the asymptotics as desired by removing the offending $\cos (\beta r) / r$ piece of the metric function. However, it is not clear that at the full non-linear level we will be able to remove the oscillations completely. Simple numerical experiments suggest that the oscillations in the bulk of the metric (for finite $r$ ) persist after the certain field redefinitions. It remains an open problem to determine the precise field redefinition for the problem at hand at the non-linear level and check their effect on the physical metric. We hope to return to this issue at a later stage.

\section{Acknowledgments}

It is a pleasure to thank Mina Aganagic, Raphael Bousso, Bernard de Wit, Atish Dabholkar, Sumit Das, Gary Horowitz, Jonathan Hsu, Shamit Kachru, Renata Kallosh, Ashoke Sen, Al Shapere, Steve Shenker and Marco Zagermann for discussions. VH and MR would in addition like to thank Atish Dabholkar for an extremely stimulating seminar at Berkeley. VH and MR are supported by the funds from the Berkeley Center for Theoretical Physics, DOE grant DE-AC03-76SF00098 and the NSF grant PHY-0098840, and in part 
by National Science Foundation under Grant No. PHY99-07949. AM is supported by the Department of Energy, under contract DE-AC02-76SF00515. 


\section{References}

[1] A. Strominger and C. Vafa, Microscopic Origin of the Bekenstein-Hawking Entropy, Phys. Lett. B 379, 99 (1996) arXiv:hep-th/9601029].

[2] A. Sen, Extremal black holes and elementary string states, Mod. Phys. Lett. A 10, 2081 (1995) arXiv:hep-th/9504147.

[3] R. M. Wald, Black hole entropy in the Noether charge, Phys. Rev. D 48, 3427 (1993) arXiv:gr-qc/9307038.

[4] K. Behrndt, G. Lopes Cardoso, B. de Wit, D. Lust, T. Mohaupt and W. A. Sabra, Higher-order black-hole solutions in $N=2$ supergravity and Calabi-Yau string backgrounds, Phys. Lett. B 429, 289 (1998) arXiv:hep-th/9801081. G. Lopes Cardoso, B. de Wit and T. Mohaupt, Corrections to macroscopic supersymmetric black-hole entropy, Phys. Lett. B 451, 309 (1999) [arXiv:hep-th/9812082]. G. Lopes Cardoso, B. de Wit and T. Mohaupt, Deviations from the area law for supersymmetric black holes, Fortsch. Phys. 48, 49 (2000) arXiv:hep-th/9904005]. G. Lopes Cardoso, B. de Wit and T. Mohaupt, Macroscopic entropy formulae and non-holomorphic corrections for supersymmetric black holes, Nucl. Phys. B 567, 87 (2000) arXiv:hep-th/9906094. G. Lopes Cardoso, B. de Wit and T. Mohaupt, Area law corrections from state counting and supergravity, Class. Quant. Grav. 17, 1007 (2000) arXiv:hep-th/9910179].

[5] G. L. Cardoso, B. de Wit, J. Kappeli and T. Mohaupt, Supersymmetric black hole solutions with $R^{* * 2}$ interactions, arXiv:hep-th/0003157. G. Lopes Cardoso, B. de Wit, J. Kappeli and T. Mohaupt, Stationary BPS solutions in $N=2$ supergravity with $R^{* * 2}$ interactions, JHEP 0012, 019 (2000) arXiv:hep-th/0009234. G. L. Cardoso, B. de Wit, J. Kappeli and T. Mohaupt, Examples of stationary BPS solutions in $N=2$ supergravity theories with $R^{* * 2-i n t e r a c t i o n s, ~ F o r t s c h . ~ P h y s . ~ 49, ~} 557$ (2001) arXiv:hep-th/0012232.

[6] A. Dabholkar, Exact counting of black hole microstates, arXiv:hep-th/0409148.

[7] A. Dabholkar, R. Kallosh and A. Maloney, A Stringy Cloak for a Classical Singularity, arXiv:hep-th/0410076.

[8] S. Ferrara, R. Kallosh and A. Strominger, N=2 extremal black holes, Phys. Rev. D 52, 5412 (1995) arXiv:hep-th/9508072]. A. Strominger, "Macroscopic Entropy of $N=2$ Extremal Black Holes," Phys. Lett. B 383, 39 (1996) arXiv:hep-th/9602111. S. Ferrara and R. Kallosh, Supersymmetry and Attractors, Phys. Rev. D 54, 1514 (1996) arXiv:hep-th/9602136. S. Ferrara and R. Kallosh, Universality of Supersymmetric Attractors, Phys. Rev. D 54, 1525 (1996) arXiv:hep-th/9603090].

[9] H. Ooguri, A. Strominger and C. Vafa, Black hole attractors and the topological string, arXiv:hep-th/0405146.

[10] C. Vafa, Black holes and Calabi-Yau threefolds, Adv. Theor. Math. Phys. 2, 207 (1998) arXiv:hep-th/9711067. 
[11] A. Sen, How Does a Fundamental String Stretch its Horizon?, arXiv:hep-th/0411255.

[12] T. Mohaupt, "Black hole entropy, special geometry and strings," Fortsch. Phys. 49, 3 (2001) arXiv:hep-th/0007195.

[13] W. A. Sabra, Black holes in $N=2$ supergravity theories and harmonic functions, Nucl. Phys. B 510, 247 (1998) arXiv:hep-th/9704147.

[14] W. A. Sabra, General static $N=2$ black holes, Mod. Phys. Lett. A 12, 2585 (1997) arXiv:hep-th/9703101.

[15] A. Borde, Geodesic focusing, energy conditons, and singularities Class. Quantum. Grav. 4, 343 (1987).

[16] G. T. Horowitz and J. Polchinski, A correspondence principle for black holes and strings, Phys. Rev. D 55, 6189 (1997) arXiv:hep-th/9612146. 